\title{
Sigmoid Volvulus during Pregnancy: A Diagnostic Trap
}

Imane Benchiba*, Meimouna Mohamed Lemine, Nissrine Mamouni, Sanaa Errarhay, Chahrazed Bouchikhi, Abdelaziz Banani

Obstetrics and Gynecology I - HASSAN II University Hospital - Fez- Morocco

DOI: 10.36348/SIJOG.2019.v02i12.002 $\quad$ | Received: 21.10.2019| Accepted: 28.10.2019| Published: 17.12 .2019

*Corresponding author: Imane Benchiba

\section{Abstract}

Colonic obstruction due to sigmoid colon volvulus during pregnancy is a rare but complication with significant maternal and fetal mortality. That requires prompt surgical intervention (decompression) to avoid intestinal ischemia and perforation. We report the case of a 38-week pregnant woman with abdominal pain and subsequent development of constipation. Preoperative diagnosis was achieved using ultrasonography: the large bowel distension and a typical whirl sign - near a sigmoid colon transition point - suggested the diagnosis of sigmoid volvulus. The decision to refer the patient for emergency laparotomy was adopted without any ionizing radiation exposure, and the pre-operative diagnosis was confirmed after surgery. Sigmoid volvulus complicating pregnancy is a rare, non-obstetric cause of abdominal pain.

Keywords: Volvulus Sigmoid, Obstruction, Pregnancy, Computed tomography (CT).

Copyright @ 2019: This is an open-access article distributed under the terms of the Creative Commons Attribution license which permits unrestricted use, distribution, and reproduction in any medium for non-commercial use (NonCommercial, or CC-BY-NC) provided the original author and source are credited.

\section{INTRODUCTION}

Intestinal obstruction in pregnancy is a rare but serious complication with significant maternal and fetal mortality, often due to delay in both diagnosis and treatment $[1,4]$. The incidence of intestinal obstruction in pregnancy ranges from 1 in 1500 to 1 in 66431 deliveries. Sigmoid volvulus is the most common cause of intestinal obstruction during pregnancy after flange occlusion, accounting for 25 to $44 \%$ of cases [2, 3]. The delay of the diagnosis is related to the lack of specificity of the functional signs and the fear of the fetal irradiation by the abdominal CT. The presenting signs/symptoms seen in these patients are the same as with non-pregnant patients and often unspecific and a high level of suspicion is essential for early diagnosis.

\section{CASE REPORT}

We describe a case of sigmoid volvulus in a patient with 38 weeks of gestation that developed complete necrosis of the left colon. Woman was admitted with 3 days of abdominal distention, vomit, and the stoppage of the passage of gases and feces. The pain was gradually increasing in severity. She had no significant past medical or surgical history. Her menstrual and antenatal history was uneventful thus far.
She was admitted and managed conservatively for these complaints at two different hospitals, with a diagnosis of delivery. The patient was evaluated by the surgical and obstetric teams in the emergency department. On physical examination she appeared dehydrated with acute respiratory distress syndrome, her abdomen was asymmetrically distended with tenderness all over. The rectum was empty on digital examination. Abdominal radiography showed severe dilation of the colon with horseshoe signal suggesting a sigmoid volvulus, pneumoperitoneum and we could not we could not identify fetal heartbeats. With a diagnosis of complicate sigmoid volvulus she was underwent to the laparotomy, The abdomen was accessed through a midline laparotomy, the obstetric team's decision was to perform a concomitant caesarean section to deliver the foetus and explore the abdomen where we found an enormously distended sigmoid loop was found with ischemic and gangrenous changes. There were no signs of perforation (Fig-1). Faced with the gangrenous sigmoid colon, resection was performed (Fig-2) followed by a primary anastomosis intra-operatively. The patient had an uneventful postoperative course thereafter and was discharged home on the 9th postoperative day on a regular diet on the 15 th day. 


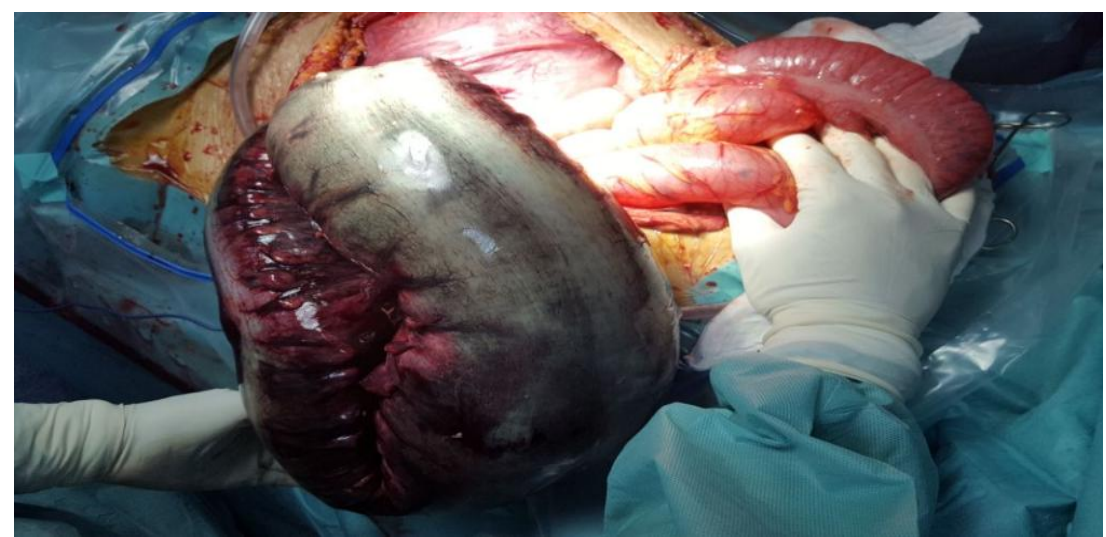

Fig-1: Dilated ischemic Gangrenous colon which had undergone volvulus displaced posteriorly by the gravid uterus

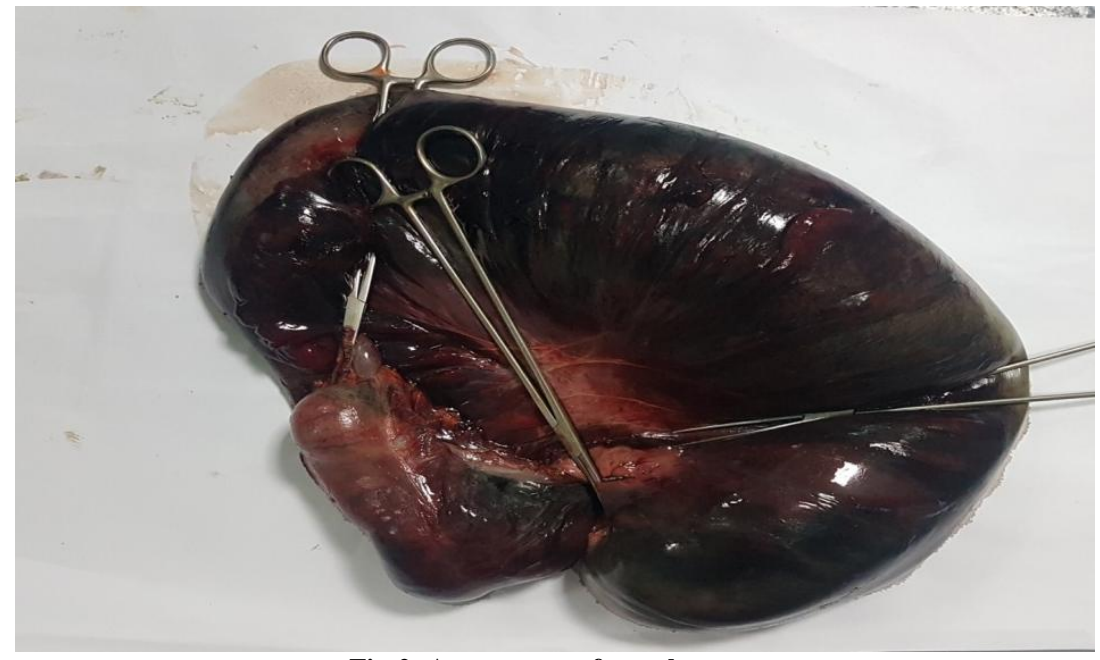

Fig-2: Appearance after colectomy

\section{DISCUSSION}

The incidence of intestinal obstruction in pregnancy ranges from 1 in 1500 to 1 in 66431 deliveries. Common causes of gestational intestinal obstruction include adhesions, volvulus, intussusceptions, hernia and appendicitis [1]. Sigmoid volvulus is the most common cause of intestinal obstruction during pregnancy after flange occlusion, accounting for 25 to $44 \%$ of cases [2-4]. In the literature the duration between onset of symptoms and presentation ranges from $1 \mathrm{~h}$ to 6 days. The mean duration is $48 \mathrm{~h}$ [5-7] sigmoid volvulus is usually reported in institutionalized, debilitated, or chronically constipated patients who have long redundant sigmoid colons [2]. A high incidence reported in Africa has been attributed to the high-fiber vegetable diet indigenous to that population [8] Harer and Harer surmised that this may be due to the increasing size of the uterus elevating a mobile sigmoid colon from the pelvis and producing a partial obstruction either due to pressure or kinking of the bowel [11]. Another study showed that in approximately $25 \%$ of cases, obstruction is the result of volvulus, usually affecting the large bowel $[9,10]$. In our cases there was no history of previous operation and there were no adhesions during operation. Obstruction was secondary to volvulus.
The diagnosis of bowel obstruction in pregnancy is often delayed because the symptoms mimic typical pregnancy-associated complaints [4]. The classical signs of bowel strangulation such as vomiting, distention and constipation, can be diminished or even absent [13]. The abdominal pain may be mild in nature and may cause colic, and may become constant and severe due to vascular compromise [14]. The first challenge lies in clinical examination. The gravid uterus limits proper physical examination of various abdominal regions. The stretched anterior abdominal wall is less sensitive to parietal irritation and may mask signs of an acute abdomen [19]. A detailed ultrasound examination may help in the differential diagnosis. Plain abdominal films demonstrate typical patterns of obstruction in $91 \%$ of the cases. At the usual dose of $0.001 \mathrm{~Gy}$ per film, even serial films obtained for follow up of the patients with suspected bowel obstruction, carries negligible risk for the fetus in the third trimester $[2,12]$. On the biological level, the most constant element is leukocytosis but its interpretation is tricky since physiological leukocytosis is common in the last two quarters $[7,12,13,16]$.

Computed tomography is the gold standard in the exploration of occlusive syndromes. Abdominal Xray for IO (intestinal obstruction) in pregnancy may be 
an acceptable and cost effective diagnostic modality [19]. Plain abdominal X-ray can identify up to $91 \%$ of typical patterns of IO $[18,19]$. Ultrasound examination can confirm the dilatation of the sigmoid colon and identify the transition point. It also confirms the presence of free fluids in the abdominal cavity and the viability of the fetus. MRI, a non-ionizing radiation modality, is also reported by some authors to be helpful in diagnosing SV during pregnancy. Low colonoscopy is an alternative to imaging and allows both confirmation of the diagnosis and eventual endoscopic detorsion. It may reveal complete occlusion of the sigmoid by highlighting oedematous mucosa or signs of ischemia but remains limited in relation to the risk of perforation [23].

The signs of severity to be investigated are a frank inflammatory syndrome with increased CRP, hyperkalemia, signs of metabolic acidosis or coagulation disorders [6].

The treatment of bowel obstruction in pregnant women is similar to that of nonpregnant women. Choice of treatment depends on the duration of pregnancy and the state of the sigmoid colon. The management in pregnancy requires a multidisciplinary approach involving general surgeon, obstetrician and neonatologist. The basis of therapy is timely surgery [4] with nasogastric decompression, fluids and electrolytes correction. Tocolytics should be used if uterine irritability is observed, and steroids initiated to promote foetal lung maturity [21]. Following initial stabilization of the patient's condition [5].

In cases with good colonic vascularization without peritoneal signs or intestinal signs of ischemia an acceptable treatment could be decompression of the volvulus, to restore canalization and adequate blood supply; detorsion could be effected by endoscopic placement of a soft rectal tube or during laparotomy. Presence or suspicion of bowel gangrene or perforation, due to prolonged alteration of vascular supply, requires urgent resection. Surgery should be performed via midline vertical incision. preferably through midline laparotomy to provide good exposure with minimal manipulation of the gravid uterus However laparoscopy during a pregnancy is technically feasible, but rather at the earliest [22]. In the third trimester, if adequate intestinal exposure cannot be obtained, caesarean section must be performed [17]. The entire bowel should be examined for other areas of obstruction. Bowel viability should be assessed carefully and segmental resection with (Hartmann's procedure) or without anastomosis is often necessary [3, 4]. Some authors prefer to perform a primary anastomosis with or without colonic washout intra-operatively when there is no contamination of the peritoneal cavity [12]. However, primary anastomosis of an unprepared distended paretic and edematous colon is generally avoided as it carries more risks to both mother and foetus. It is worth mentioning that early diagnosis would make resection and primary anastomosis a safe approach, with the distinct advantage of a reduced hospital stay and avoidance of further surgery [12, 20].

In addition to the vitality of intestinal wall, Alshawi et al., proposed the following management options based on the stage of pregnancy: in the first trimester, and in recurrent cases in the second trimester, colonoscopic detorsion and rectal decompression are performed Sigmoid colectomy is recommended in the second trimester, because endoscopic decompression is associated with high recurrence rate of volvulus, and operating in the second trimester is associated with lower miscarriage rate than the first [20]. In the third trimester, endoscopic decompression is performed until fetal maturity, when elective labor followed by sigmoidectomy provides the final treatment. However, in later pregnancy, endoscopic derotation could be limited by enlargement of the uterus, and surgical derotation or sigmoidectomy are often chosen as treatment: resection could be eventually postponed in the post-partum in cases of viable tissue [2].

When intestinal obstruction complicates pregnancy, both mother and fetus are at risk [4]. In a review on intestinal obstruction during pregnancy in 1937 , it has been reported that the maternal mortality rate was 21 per cent and the fetal mortality rate was 50 per cent [1] Foetal death can result from a reduction of placental blood flow in the hypovolemia, or increased intra abdominal pressure as a result of massive sigmoid dilatation [18]. From these figures, only early diagnosis and timely surgical intervention can improve both maternal and foetal outcomes.

\section{CONCLUSION}

Sigmoid volvulus is a rare and potentially serious complication during pregnancy. Diagnosis in pregnancy requires a high index of suspicion in a patient who presents with complaints of abdominal pain and evidence of bowel obstruction. Prompt intervention is necessary to minimize maternal and fetal morbidity and mortality. Surgical treatment is essential in most cases.

Conflicts of Interest: None of the authors have any conflicts of interest to declare.

Consent: Written informed consent was obtained from the patient prior to the writing of the case report.

\section{REFERENCE}

1. Perdue, P. W., Johnson, H. W., \& Stafford, P. W. (1992). Intestinal obstruction complicating pregnancy. The American Journal of Surgery, 164(4), 384-388. 
2. Ballantyne, G. H., Brandner, M. D., Beart Jr, R. W., \& Ilstrup, D. M. (1985). Volvulus of the colon. Incidence and mortality. Annals of surgery, 202(1), 83-92.

3. Coughlan, B. M. (1978). Acute intestinal obstruction during pregnancy.JR Coll Surg Edinb, 23, 175-177.

4. Redlich, A., Rickes, S., Costa, S. D., \& Wolff, S. (2007). Small bowel obstruction in pregnancy. Archives of Gynecology and Obstetrics, 275(5), 381-383.

5. Aftab, Z., Toro, A., Abdelaal, A., Dasovky, M., Gehani, S., Mola, A. A., \& Di Carlo, I. (2014). Endoscopic reduction of a volvulus of the sigmoid colon in pregnancy: case report and a comprehensive review of the literature. World Journal of Emergency Surgery, 9(1), 41.

6. Khan, M. R., \& Ur Rehman, S. (2012). Sigmoid volvulus in pregnancy and puerperium: a surgical and obstetric catastrophe. Report of a case and review of the world literature. World Journal of Emergency Surgery, 7(1), 10.

7. Ribeiro Nascimento, E. F., Chechter, M., Fonte, F. P., Puls, N., Valenciano, J. S., Fernandes Filho, C. L. P., ... \& Martinez, C. A. R. (2012). Volvulus of the sigmoid colon during pregnancy: a case report. Case reports in obstetrics and gynecology, 2012.

8. Elmasri, S. H., \& Khalil, T. (1976). Volvulus of the sigmoid in Khartoum, Sudan. Tropical and geographical medicine, 28(4), 297-302.

9. Connolly, M. M., Unti, J. A., \& Nora, P. F. (1995). Bowel obstruction in pregnancy. Surgical Clinics of North America, 75(1), 101-113.

10. Mirza, M. S., Mulla, M., \& Hall, R. I. (2009). Large bowel obstruction in pregnancy: a rare entity, an unusual cause. Archives of gynecology and obstetrics, 279(2), 177-178.

11. HARER, W. B. (1958). Volvulus complicating pregnancy and puerperium: report of three cases and review of literature. Obstetrics \& Gynecology, 12(4), 399-406.

12. Machado, N. O., \& Machado, L. S. (2009). Sigmoid volvulus complicating pregnancy managed by resection and primary anastomosis: case report with literature review. Sultan Qaboos University medical journal, 9(1), 84-88.

13. J. Kusnetzoff, AD Barata, C. Casalnuovo, LM Alvarez, D. (1997). Massive midgut volvulus during pregnancy. Journal of Obstetrics and Gynaecology, 17(6), 583-583.
14. Ventura-Braswell, A. M., Satin, A. J., \& Higby, K. (1998). Delayed diagnosis of bowel infarction secondary to maternal midgut volvulus at term. Obstetrics \& Gynecology, 91(5), 808-810.

15. Lord, S. A., Boswell, W. C., \& Hungerpiller, J. C. (1996). Sigmoid volvulus in pregnancy. The American surgeon, 62(5), 380-382.

16. Kolusari, A., Kurdoglu, M., Adali, E., Yildizhan, R., Sahin, H. G., \& Kotan, C. (2009). Sigmoid volvulus in pregnancy and puerperium: a case series. Cases Journal, 2(1), 9275.

17. Allen JR, Helling TS, Langenfeld M: Intraabdominal surgery during pregnancy. Am J Surg. 1989, 158: 567-569. 10.1016/00029610(89)90194-3.

18. Vo, T. M., Gyaneshwar, R., \& Mayer, C. (2008). Concurrent sigmoid volvulus and herniation through broad ligament defect during pregnancy: case report and literature review. Journal of Obstetrics and Gynaecology Research,34(4pt2), 658-662.

19. Zachariah, S. K., \& Fenn, M. G. (2014). Acute intestinal obstruction complicating pregnancy: diagnosis and surgical management. BMJ case reports, 2014, bcr2013203235.

20. Alshawi, J. S. (2005). Recurrent sigmoid volvulus in pregnancy: report of a case and review of the literature. Diseases of the colon \& rectum, 48(9), 1811-1813.

21. Twité, N., Jacquet, C., Hollemaert, S., El, F. I., Dumont, G., Nasr, A., ... \& Busine, A. (2006). Intestinal obstruction in pregnancy. Revue medicale de Bruxelles, 27(2), 104-109.

22. Azuar, A. S., Bouillet-Dejou, L., Jardon, K., Lenglet, Y., Canis, M., Bolandard, F., ... \& Lemery, D. (2009). Laparoscopy during pregnancy: experience of the French university hospital of Clermont-Ferrand. Gynecologie, obstetrique \& fertilite, 37(7-8), 598-603.

23. Dray, X., Hamzi, L., Dico, R. L., \& Barranger, E. (2012). Endoscopic reduction of a volvulus of the sigmoid colon in a pregnant woman. Digestive and Liver Disease, 44(5), 447.

24. Sascha Dua, R., Rothnie, N. D., \& Gray, E. A. (2007). Sigmoid volvulus in the puerperium. International Journal of Gynecology \& Obstetrics, 97(3), 195-210.

25. Shenhav, S., Gemer, O., Segal, S., Linova, L., \& Joffe, B. (2000). Preoperative diagnosis of intestinal intussusception in pregnancy. A case report. The Journal of reproductive medicine, 45(6), 501-503. 